\title{
Delineamento dos casos de lesão renal de um hospital privado do interior de São Paulo
}

\author{
Outline of renal injury cases in a private hospital in a city of São Paulo \\ Resumen de casos de lesiones renales en un hospital privado en el interior de São Paulo
}

Recebido: 22/03/2021 | Revisado: 28/03/2021 | Aceito: 31/03/2021 | Publicado: 10/04/2021

\author{
Mariana Paula Rodrigues Costa \\ ORCID: https://orcid.org/0000-0002-1072-4606 \\ Centro Universitário da Fundação Hermínio Ometto, Brasil \\ E-mail:maripaula85@gmail.com. \\ Juliana Furlan Ravagnani \\ ORCID: https://orcid.org/0000-0002-2058-1258 \\ Universidade Estadual de Campinas, Brasil \\ E-mail: jravagnani@hc.unicamp.br \\ Aline Scharr Rodrigues \\ ORCID: https://orcid.org/0000-0002-8336-6037 \\ Universidade de São Paulo, Brasil \\ E-mail: alinescharr@usp.br \\ Clarice Santana Milagres \\ ORCID: https://orcid.org/0000-0002-9164-4340 \\ Centro Universitário da Fundação Hermínio Ometto, Brasil \\ E-mail:claricemilagres01@gmail.com
}

\begin{abstract}
Resumo
A Doença Renal Crônica é caracterizada como uma Doença Crônica Não Transmissível que atinge proporções mundiais. Fatores que contribuem para o avanço desse quadro, é o aumento da incidência das doenças de base como Hipertensão Arterial Sistêmica (HAS) e Diabetes mellitus (DM) e a elevada ocorrência de Injúria Renal Aguda em ambientes intra-hospitalares. Objetivo: identificar o quadro dos pacientes com lesão renal que realizaram tratamento hemodialítico em um hospital privado do interior de São Paulo. Métodos: estudo transversal, retrospectivo, descritivo, com abordagem quantitativa, realizado através da análise de prontuários eletrônicos de um hospital particular, entre os meses de janeiro a junho de 2017. Realizou-se análise estatística exploratória, com análise de frequência dos dados obtidos. Resultados: foram analisados 58 prontuários. A média da idade identificada foi 70,7 anos $( \pm 15,5)$, com mínima de 28 anos e máxima de 98 anos. Quanto às condições de saúde, 63,7\% apresentavam história pregressa de Hipertensão Arterial Sistêmica, enquanto metade da amostra apresentava cardiopatias e o Diabetes mellitus foi constatado em $41,3 \%$ da amostra. O diagnóstico inicial relacionado às doenças cardiovasculares correspondeu a 32,7\% dos dados, sepses a $20,6 \%$ e as insuficiências renais estiveram presentes em 8,6\% destes diagnósticos. Em relação à Terapia Renal Substitutiva, aproximadamente $80 \%$ possuíam Doença Renal Aguda, predominando o acesso vascular temporário de triplo lúmen. Conclusão: identificar os fatores que contribuem com o quadro de DRC intra-hospitalar oportuniza melhor condição de salvaguardar a vida dos pacientes, assim como possibilita a identificação precoce de fatores de risco de piora da função renal.
\end{abstract}

Palavras-chave: Lesão renal aguda; Diálise; Registros eletrônicos de saúde.

\begin{abstract}
Chronic Kidney Disease is characterized as a Chronic Non-Communicable Disease that reaches worldwide proportions. Factors that contribute to the advancement of this situation are the increased incidence of basic diseases such as Systemic Arterial Hypertension (SAH) and Diabetes mellitus (DM) in the world population and the high occurrence of Acute Kidney Injury in in-hospital settings. Objective: To identify the situation of patients with injury renal who underwent hemodialysis in a private hospital in the interior of São Paulo. Methods: a cross-sectional, retrospective, descriptive study, with a quantitative approach, carried out through the analysis of electronic medical records of a private hospital, between January to June 2017. An exploratory statistical analysis was carried out, with a frequency analysis of the data obtained. Results: 58 medical records were analyzed. The average age identified was 70,7 years $( \pm 15,5)$, with a minimum of 28 years and a maximum of 98 years. As for health conditions, 63,7\% had a history of Systemic Arterial Hypertension, while half of the sample had heart disease and Diabetes mellitus was found in $41,3 \%$ of the sample. The initial diagnosis related to cardiovascular diseases corresponded to $32,7 \%$ of the data, sepsis to $20,6 \%$, and renal insufficiencies were present in $8,6 \%$ of these diagnoses. Regarding Renal Replacement Therapy, approximately $80 \%$ had Acute Kidney Disease, with temporary triple-lumen vascular access predominating. Conclusion: identifying the factors that contribute to the picture of in-hospital CKD provides a better condition to safeguard patients' lives, as well as enabling the early identification of risk factors for worsening renal function.
\end{abstract}

Keywords: Acute kidney injury; Dialysis; Electronic health records. 


\begin{abstract}
Resumen
La enfermedad renal crónica se caracteriza por ser una enfermedad crónica no transmisible que alcanza proporciones mundiales. Los factores que contribuyen al avance de esta situación son la mayor incidencia de enfermedades básicas como la Hipertensión Arterial Sistémica (HSA) y la Diabetes mellitus (DM) y la alta ocurrencia de Lesión Renal Aguda en ambientes intrahospitalarios. Objetivo: identificar el estado de los pacientes con daño renal sometidos a hemodiálisis en un hospital privado del interior de São Paulo. Métodos: estudio descriptivo, transversal, retrospectivo, con abordaje cuantitativo, realizado a través del análisis de historias clínicas electrónicas de un hospital privado, entre los meses de enero a junio de 2017. Se realizó un análisis estadístico exploratorio, con análisis de frecuencia de los datos obtenidos. Resultados: se analizaron 58 historias clínicas. La edad promedio identificada fue de 70,7 años $( \pm 15,5)$, con un mínimo de 28 años y un máximo de 98 años. En cuanto a las condiciones de salud, el 63,7\% tenía antecedentes de Hipertensión Arterial Sistémica, mientras que la mitad de la muestra tenía enfermedad cardíaca y se encontró Diabetes mellitus en el 41,3\% de la muestra. El diagnóstico inicial relacionado con enfermedades cardiovasculares correspondió al 32,7\% de los datos, la sepsis al 20,6\% e insuficiencia renal se presentó en el 8,6\% de estos diagnósticos. En cuanto a la Terapia de Reemplazo Renal, aproximadamente el 80\% tenía Enfermedad Renal Aguda, con predominio del acceso vascular temporal de triple lumen. Conclusión: identificar los factores que contribuyen al cuadro de la ERC intrahospitalaria proporciona una mejor condición para salvaguardar la vida de los pacientes, además de permitir la identificación precoz de los factores de riesgo de empeoramiento de la función renal.
\end{abstract}

Palabras clave: Lesión renal; Diálisis; Registros electrónicos de salud.

\title{
1. Introdução
}

A Doença Renal Crônica (DRC) ocorre a partir da perda progressiva e irreversível da função renal. Assim, os rins perdem sua função glomerular, tubular e endócrina, não conseguindo conservar seu funcionamento. Os rins são órgãos fundamentais para a manutenção da homeostase do corpo humano. A diminuição progressiva da função renal implica em um comprometimento de todos os outros órgãos. Dessa forma, a DRC pode ser caracterizada pela deterioração das funções bioquímicas e fisiológicas de todos os sistemas do organismo, secundária ao acúmulo de catabólitos (toxinas urêmicas), alterações do equilíbrio hidroeletrolítico e ácido-básico, por exemplo, acidose metabólica; hipovolemia; hipercalemia; hiperfosfatemia; anemia e distúrbio hormonal hiperparatireoidismo, infertilidade e retardo no crescimento (Bacallao-Mendez, Mañalich-Comas \& GalvizuDías, 2016; Dias, Silva, Maia, Morais, Silva \& Oliveira, 2017).

Segundo o censo brasileiro de diálise da Sociedade Brasileira de Nefrologia (SBN) em 2019, a distribuição de pacientes em hemodiálise (HD) foi um pouco mais de 93\%, enquanto o restante encontrava-se em diálise peritoneal (DP). A estimativa de incidência anual de novos pacientes com doença renal em diálise no país, está em torno de 42.500, com tendências a aumentar. Calcula-se também que mais de 130 mil pacientes estejam em tratamento dialítico e os gastos para manutenção dos programas de Terapia Renal substitutiva (TRS), como hemodiálise, diálise peritoneal e transplante renal, consumam mais de dois bilhões de reais por ano, correspondente a mais de 10\% de todo o orçamento do Ministério da Saúde. Do total desses serviços, 79\% são financiados pelo Sistema único de Saúde (SUS) e os outros 21\% resultam de convênios (Neves, Sesso, Thomé, Lugon \& Nascimento, 2020). Vale ressaltar as informações apresentadas são referentes à Doença Renal Crônica, e não estão inseridos os dados relacionados aos pacientes com injúria renal aguda (IRA), assim como sua progressão para a cronicidade (Neves et al., 2020; Thomé, Sesso, Lopes, Lugon \& Martins, 2019).

Em relação à IRA, esta é definida como um rápido declínio da Taxa de Filtração Glomerular (TFG), no qual o processo de lesão renal aguda ocasiona diversas alterações em nível celular e molecular que, finalmente, levam a uma disfunção renal e lesão estrutural. Considerada um importante problema de saúde, a IRA apresenta altas taxas de incidência, particularmente no ambiente intrahospitalar, sendo responsável por $1 \%$ de todas as internações hospitalares, complicando em $7 \%$ delas e sua ocorrência aumenta para 40-60\% em pacientes internados em Unidade de Terapia Intensiva (UTI). Apesar dos avanços significativos da nefrologia e dos cuidados intensivos, a taxa de mortalidade de pacientes hospitalizados com IRA permanece relativamente constante, em torno de 50\% nas últimas décadas. A identificação das diversas etiologias adquiridas na comunidade que induzem à IRA já foram identificadas, estando entre elas a isquemia, sepse e toxinas (inclusive medicamentos), comuns em 
pacientes hospitalizados. Acrescenta-se que a creatinina continua a ser o padrão laboratorial para o diagnóstico da IRA(GonzálezMilán, Escalna-Gonzáles \& Pavón-Rojas, 2020).

A IRA é uma das complicações mais comuns em ambiente hospitalar e sua incidência acontece conforme a gravidade do paciente. É uma patologia reversível, caracterizada pela rápida queda da capacidade dos rins em retirar as escorias do organismo, o que pode levar a distúrbios hídricos, eletrólitos e acidobásicos. As causas são diversas, entre elas, a hipoperfusão, causada principalmente por sepses ou hipovolemia, e o uso prolongado de nefrotóxicos. Estes eventos provocam lesões que causam a diminuição rápida da função dos néfrons, levando a IRA e que, se não reverter rapidamente, pode tornar-se irreversível ou levar o paciente ao óbito (Bacallao Mendez et al., 2016; González-Milán et al., 2020; Vanmassenhove, Kieltein, Jörres \& Biesen, 2017).

Essa insuficiência pode se manifestar de várias formas, como a sede, a hipotensão ortostática, redução de pressão venosa jugular, sinais de desidratação, sedimento urinário ativo, edema, hipertensão e oligúria, entre outros. De uma forma geral as indicações para reverter a IRA é a Terapia Renal Substitutiva (TRS), sendo a diálise, o processo artificial de remoção de solutos e fluidos em excesso cujos rins em falência não são capazes de excretar (Carlino et al., 2020; Rodríguez-Palomares, García, Cañamas \& Arriba, 2015).

Devido à elevada incidência de doença renal em ambientes intra hospitalares, mesmo com os avanços das tecnologias existentes em nível terciário, ainda há expressivo número de indivíduos que progridem para a cronificação da injuria renal aguda. Conhecer os fatores predisponentes relacionados ao desenvolvimento dos agravos são relevantes à medida que norteiam planejamento dos cuidados clínicos e terapêuticos, reduzindo assim, as complicações e preservando vidas. Diante do exposto, este estudo buscou identificar o quadro dos pacientes com lesão renal que realizaram tratamento hemodialítico em um hospital privado do interior de São Paulo.

\section{Metodologia}

\section{Delineamento}

Trata-se de um estudo transversal, retrospectivo e descritivo com abordagem quantitativa. A pesquisa descritiva tem a finalidade de observar, descrever e documentar os aspectos da situação. Esse tipo de estudo envolve a coleta de dados em um ponto específico de tempo. Os delineamentos transversais são apropriados para descrever a situação, o status do fenômeno ou as relações entre o fenômeno e um ponto fixo(Marcus, MT \& Liehr PR., 2001).

\section{Local da Pesquisa}

A pesquisa aconteceu em um hospital privado do interior de São Paulo. Trata-se de um hospital geral de alta complexidade, de médio porte e conta com 19 leitos de Unidade de Tratamento Intensivo (UTI) adulto, oito leitos de UTI pediátrico e 69 leitos de enfermaria. O hospital possui um serviço de Nefrologia desde o ano 2000 e no qual equipe deste serviço é responsável pela assistência aos pacientes com doença renal, seja IRA ou DRC em TRS.

\section{Coleta de dados e amostra}

Foi realizado um levantamento retrospectivo de prontuários eletrônicos dos pacientes internados entre os meses de janeiro à junho de 2017. A busca de dados foi realizada pelo Sistema de Dados do local por meio da seleção das prescrições que utilizavam a palavra hemodiálise. Desta forma, foram identificadas somente e exclusivamente, pacientes neste hospital que realizaram tratamento hemodialítico no período proposto, assim como as demais informações referentes a este tratamento dialítico. Todos os dados foram coletados pela pesquisadora, através de um instrumento estruturado, constando dados sociodemográficos, de condições de saúde e referentes à hemodiálise. 
Foi realizado um prévio contato com o responsável por todos os setores (enfermarias e UTI's), com apresentação e esclarecimento sobre a pesquisa. Após as devidas autorizações, a coleta de dados retrospectivos ocorreu em janeiro e fevereiro de 2018, com tempo estimado de aproximadamente duas horas para cada indivíduo encontrado no banco de dados e devida inserção de informações completas no instrumento criado.

Nessa pesquisa foram analisados os prontuários eletrônicos via Sistema MVpep ${ }^{\circledR}$. Segundo a Organização Mundial da Saúde (OMS), as definições de Electronic Medical Record (EMR) ou Prontuário Médico Eletrônico (PME), são usadas para descrever sistemas desenvolvidos para consultórios médicos ou centros de saúde que incluem elementos de identificação do paciente, medicamentos e geração de receitas; registros de resultados de exames laboratoriais e, em alguns casos, todas as informações de saúde registradas pelo médico em cada visita do paciente. O Computer-based Patient Record (CPR), ou Registro do Paciente Baseado no Computador, é mais focado nos registros hospitalares(Patrício, Machiavelli, Maia \& Navaes, 2011).

Como critérios de inclusão, foram elencados os prontuários de pacientes que realizaram tratamento hemodialítico neste hospital privado do interior de São Paulo nos primeiros seis meses de 2017. Quanto aos critérios de exclusão, estavam aqueles prontuários de pacientes que neste mesmo período de janeiro a junho de 2017 não realizaram tratamento hemodialítico neste mesmo local.

\section{Autorizações e procedimentos éticos}

Fora requisitado a superintendência do hospital em questão, para autorização da coleta de dados, assim como o projeto foi encaminhado ao Comitê de Ética em Pesquisa (CEP) do Centro Universitário da Fundação Hermínio Ometto/FHO e a Plataforma Brasil, apresentando CAAE n 83213918.1.0000.5385 e aprovação nº 2.886.839.

Após aprovação ética e conforme previsto na Resolução do Conselho Nacional de Saúde (CNS) 466/2012, os dados foram coletados por meio do prontuário eletrônico e por se tratar de pesquisa retrospectiva com uso de prontuários, solicitou-se e foi concedido dispensa do Termo de Consentimento Livre e Esclarecido (TCLE). Mantendo-se o anonimato dos sujeitos e a confidencialidade dos dados coletados para pesquisa.

\section{Variáveis do estudo}

As variáveis analisadas foram representadas pelos dados sociodemográficos, condições de saúde e tratamento hemodialítico.

Variáveis sociodemográficos: faixa etária (até 30 anos, 31-59 anos e 60 anos ou mais), sexo (masculino e feminino), etnia (branca, negra e parda), escolaridade (até 3 anos, 4 a 9 anos, 9 anos e mais) e estado civil (casado, separado/divorciado, solteiro e viúvo).

Variáveis das condições de saúde: informações de história pregressa de doenças, sendo elas: hipertensão arterial sistêmica, diabetes tipo 1 ou 2, depressão, cardiopatias, neuropatias e neoplasias (não e sim).

Variáveis hemodialíticas: diagnóstico inicial (sepse, doenças cardiovasculares, doenças neurológicas, doenças respiratórias, insuficiências renais, infecções agudas e outras), entrada no serviço de Terapia Renal Substitutiva (doença renal aguda e doença renal crônica), acesso vascular (fistula arteriovenosa, cateter de triplo lúmen, cateter de duplo lúmen), transplante renal prévio (não e sim), em caso de transplante, tipo de doador (vivo e falecido).

\section{Análise de dados}

Para o armazenamento dos dados foi utilizado o software Office, Excel versão 2013 e para análise de dados foi utilizado o software Stata versão 9.0. Foi empregada a análise descritiva absoluta e de frequência, \pm desvio padrão, para as variáveis quantitativas. Os dados foram apresentados em tabelas e agrupados conforme paridades de temas. 


\section{Resultados}

Foram identificados 58 prontuários de pacientes que realizaram tratamento hemodialítico no referido hospital. A média da idade identificada foi 70,7 anos $( \pm 15,5)$. A idade mínima encontrada foi 28 anos, enquanto a maior idade foi 98 . Houve predomínio da faixa etária de 60 anos ou mais, correspondendo a aproximadamente $76 \%$ da amostra encontrada nos prontuários.

De acordo com a Tabela 1, nos prontuários analisados, houve predomínio do sexo feminino $(56,9 \%)$, brancos $(92,4 \%)$, escolaridade entre 4 e 9 anos $(67,2 \%)$ e casados $(48,2 \%)$.

Tabela 1. Análise descritiva de variáveis sociodemográficas em prontuários eletrônicos dos pacientes de um hospital privado do interior de São Paulo, junho 2017.

\begin{tabular}{lll}
\hline Variáveis/Categorias & n* & Prevalência (\%) \\
\hline Faixa etária & 1 & $1,8 \%$ \\
$\quad$ Até 30 anos & 13 & $22,4 \%$ \\
$\quad 31-59$ anos & 44 & $75,8 \%$ \\
$\quad 60$ anos ou mais & & \\
Sexo & 25 & $43,1 \%$ \\
$\quad$ Masculino & 33 & $56,9 \%$ \\
$\quad$ Feminino & & \\
Etnia & 55 & $92,4 \%$ \\
$\quad$ Branca & 1 & $1,7 \%$ \\
$\quad$ Parda & 2 & $3,4 \%$ \\
$\quad$ Amarela & & \\
Escolaridade & 1 & $1,7 \%$ \\
Até 3 anos & 39 & $67,2 \%$ \\
4 a 9 anos & 18 & $31 \%$ \\
9 anos e mais & & \\
Estado Civil & 28 & $48,2 \%$ \\
Casado & 4 & $6,9 \%$ \\
Separado/Divorciado & 7 & $12 \%$ \\
Solteiro & 19 & $32,7 \%$ \\
Viúvo & & \\
\hline
\end{tabular}

* número de indivíduos. Fonte: Autores.

Em relação às condições de saúde, 63,7\% apresentavam história pregressa de hipertensão arterial sistêmica, enquanto metade da amostra apresentava cardiopatias e o diabetes apareceu em 41,3\% da amostra. O diagnóstico inicial relacionado às doenças cardiovasculares correspondeu a 32,7\% dos dados, sepses a 20,6\%, enquanto as insuficiências renais estiveram presentes em apenas 8,6\% destes diagnósticos (Tabela 2). 
Tabela 2. Análise descritiva de variáveis das condições de saúde em prontuários eletrônicos dos pacientes de um hospital privado do interior de São Paulo, junho 2017.

\begin{tabular}{lll}
\hline Variáveis/Categorias & $\mathbf{n}^{*}$ & Prevalência (\%) \\
\hline História de hipertensão arterial sistêmica & 21 & $36,2 \%$ \\
$\quad$ Não & 37 & $63,7 \%$ \\
$\quad$ Sim & & \\
História de diabetes tipo 1 ou 2 & 34 & $58,6 \%$ \\
$\quad$ Não & 24 & $41,3 \%$ \\
$\quad$ Sim & & \\
História de depressão & 53 & $80,8 \%$ \\
$\quad$ Não & 5 & $19,2 \%$ \\
$\quad$ Sim & & \\
História de cardiopatias & 29 & $50 \%$ \\
$\quad$ Não & 29 & $50 \%$ \\
$\quad$ Sim & & \\
História de neuropatias & 52 & $89,6 \%$ \\
$\quad$ Não & 6 & $10,3 \%$ \\
$\quad$ Sim & & \\
História de neoplasias & & \\
$\quad$ Não & 48 & $82,7 \%$ \\
Sim & 10 & $17,2 \%$ \\
\hline
\end{tabular}

* número de indivíduos. Fonte: Autores.

Nas variáveis referentes à TRS, aproximadamente $80 \%$ dos prontuários foram preenchidos como presença de injúria renal aguda, predominando o acesso vascular temporário de triplo lúmen (67,8\%) e duplo lúmen (21,4\%), respectivamente. Apenas em dois prontuários houve transplante renal prévio, sendo os dois enxertos vindos de doadores falecidos (Tabela 3 ). 
Tabela 3. Análise descritiva de variáveis de hemodiálise em prontuários eletrônicos dos pacientes de um hospital privado do interior de São Paulo, junho 2017.

\begin{tabular}{lll}
\hline Variáveis/Categorias & n* & Prevalência (\%) \\
\hline Diagnóstico Inicial & 12 & $20,6 \%$ \\
$\quad$ Sepse & 19 & $32,7 \%$ \\
$\quad$ Doenças Cardiovasculares & 7 & $12 \%$ \\
$\quad$ Doenças Neurológicas & 3 & $5,1 \%$ \\
$\quad$ Doenças Respiratórias & 5 & $8,6 \%$ \\
$\quad$ Insuficiências Renais & 6 & $10,3 \%$ \\
$\quad$ Infecções Agudas & 6 & $10,3 \%$ \\
$\quad$ Outros & & \\
Entrada no serviço de TRS & 46 & $79,3 \%$ \\
$\quad$ Injúria Renal Aguda (IRA) & 12 & $20,6 \%$ \\
$\quad$ Doença Renal Crônica (DRC) & & \\
Tipo de acesso vascular & 6 & $10,7 \%$ \\
$\quad$ Fistula arteriovenosa (FAV) & 38 & $67,8 \%$ \\
Cateter de triplo lúmen (CTL) & 12 & $21,4 \%$ \\
Cateter de duplo lúmen (CDL) & & $80 \%$ \\
Transplante prévio & 8 & $20 \%$ \\
Não & 2 & \\
Sim & &
\end{tabular}

* número de indivíduos. Fonte: Autores.

\section{Discussão}

O prontuário do paciente é um documento básico em hospitais ou grandes clínicas e permeia toda a atividade inerente aos cuidados diretos ou indiretos prestados aos pacientes(Albuquerque, Albuquerque, Souza, Santos \& Rêgo, 2017; Patrício et al., 2011). No Brasil, o conceito mais utilizado para prontuário foi proposto pelo Conselho Federal de Medicina (CFM), segundo a Resolução ${ }^{\circ} 1.638$ de 2002, que define prontuário do paciente como:

"Um documento único constituído de um conjunto de informações, sinais e imagens registradas, geradas a partir de fatos, acontecimentos e situações sobre a saúde do paciente e a assistência a ele prestada, de caráter legal, sigiloso e científico, que possibilita a comunicação entre membros da equipe multiprofissional e a continuidade da assistência prestada ao indivíduo"(CFM, 2002).

Em 2007, o CFM autorizou o uso de sistemas informatizados para a guarda e registro deste tipo de informação relacionada ao paciente (CFM, 2007). O Prontuário Eletrônico de Paciente (PEP), independentemente do conceito, deve ser sigiloso, possuir caráter assistencial, ético-legal e científico; e possibilitar a comunicação entre os membros da equipe multiprofissional, assim como a continuidade da assistência prestada ao mesmo (Albuquerque et al., 2017; Araújo, Rechmann \& Magalhães, 2019; BRASIL, 2018; Schütz \& Oliveira, 2020).

Em 2019, segundo o censo da Sociedade Brasileira de Nefrologia, foram identificados 42,5\% dos pacientes em dialise com idades entre 45 a 64 anos de idade, 22,2\% com idades de 65 a 74 anos e 13,3\% daqueles com idade de 75 e mais (Neves et 
al., 2020). Na análise dos prontuários pesquisados, verificou-se um percentual expressivo de pacientes na terceira idade, indo na contrapartida dos dados epidemiológicos da DRC.

Em relação ao sexo, a presença de internações femininas foram mais prevalente, corroborando com o estudo de Fernandes et al. (2015), realizado no setor de nefrologia de um hospital universitário e em uma clínica de hemodiálise no nordeste brasileiro no qual 53\% eram mulheres (Fernandes, Silva, Dantas, Paiva, Araújo \& Lira, 2015). Segundo os autores, a feminilização na diálise deve-se ao acometimento por hipertensão arterial no sexo feminino com maior prevalência, se comparado aos homens. Esta variação pode ocorrer pelo estilo de vida de muitas delas, ao utilizar períodos prolongados de uso de anticoncepcionais orais, menopausa, tripla carga de tarefas, além dos elevados níveis de estresse com a qual são continuamente expostas. Contudo, vale mencionar, que hábitos de vida modificáveis são necessários entre ambos, seja homem ou mulher, e que descuidos alimentares como o consumo excessivo de sal e bebidas alcoólicas; aumento de peso, sedentarismo e tabagismo representam risco à saúde e ao desenvolvimento da doença renal.

Logo, quando analisadas as internações hospitalares e a doença renal, o sexo masculino é mais prevalente. Stochero et al. (2020) verificou que a prevalência de internações por nefropatia induzida por contraste (NIC) em pacientes cardíacos de um setor de hemodinâmica são em sua maioria do sexo masculino, assim como Gaspari et al. (2019) em uma revisão sobre cardiologia intensivista em relação aos procedimentos, métodos e diagnósticos que requerem o uso de contraste iodado e a NIC, apresentaram dados epidemiológicos consistentes com a presente pesquisa, no qual o sexo masculino apresenta maiores prevalências de internações e cuidados de saúde que demandam maiores complexidades e tempo de tratamentos.

O estado civil, amplamente estudado como variável social dos portadores de doença renal também foi verificada na presente pesquisa, demonstrando que a maioria daqueles que estiveram internados e apresentaram lesão renal são casados. Segundo Fernandes et al. (2020) em um estudo sobre a qualidade de vida de pacientes em TRS e a estratificação de risco da doença renal, puderam verificar que maioria dos pacientes em terapia dialítica eram casados. Segundo a literatura, a ausência de um companheiro para aqueles com doença renal pode ser danosa à continuidade dos cuidados em saúde já aprendidos, assim como aqueles cuidados que deverão ser aprendidos em caso de progressão da injuria renal aguda para a doença renal crônica.

Quanto à escolaridade, o presente trabalho encontrou informações diferenciadas em relação à literatura já consolidada, no qual portadores de doença renais apresentam baixa escolaridade, e esta pode resultar em limitações de conhecimento sobre as terapias renais substitutivas, autocuidado, tratamentos dialíticos, prevenção das doenças associadas e pior percepção da condição de saúde determinada pela internação e pela doença renal, seja esta recente ou não (Fernandes et al., 2020; Ribeiro et al., 2018). No entanto, os dados obtidos nesta pesquisa foram provenientes de um hospital privado e como tal, a amostra apresenta maiores rendas, com possibilidade de obtenção de planos de saúde privados, e, consequentemente, melhores acessos aos procedimentos diagnósticos e encaminhados aos respectivos tratamentos em tempo oportuno (Malta et al., 2017). O estudo de Malta et al. (2017) também apresenta um público de etnia caucasiana, assim como os resultados na presente pesquisa.

A doença renal pertence a um grupo de doenças não transmissíveis e degenerativas. É reconhecida mundialmente como um problema de saúde pública ao estar associada à elevados custos de tratamento, aumento no financiamento destinada aos serviços de saúde, queda da qualidade de vida e é uma das principais causas de mortalidade global (Pinho et al., 2015). Por ano, cerca de três milhões e meio de pessoas morrem por condições associadas à doença renal crônica, como nefroesclerose hipertensiva e nefropatia diabética e cerca de $10 \%$ da população mundial apresenta algum grau dessa cronicidade, advinda, também, inicialmente pela injuria renal aguda. Neste estudo foi evidenciado que aproximadamente $64 \%$ apresentavam HAS, 41,3\% diabetes e metade da amostra apresentava algum comprometimento cardiovascular. Estes achados consolidam a caraterização de indivíduos e as doenças associadas à instalação e progressão das doenças renais.

Em todo o país a necessidade de políticas públicas de saúde mais efetivas faz-se necessária para uma real prevenção da injuria renal e sua cronificação. No mais, o constante monitoramento dos indivíduos hospitalizados é fundamental para a deteç̧ão 
das fases pré-dialíticas e consequente instalação de TRS nos pacientes (Fernandes et al., 2020). Mundialmente há elevada prevalência de doenças de base como hipertensão arterial, diabetes e cardiopatias nos indivíduos hospitalizados, sendo precursoras do aparecimento das doenças renais, em especial, a injuria renal aguda. Ponce et al. (2011) verificaram que no diagnóstico inicial dos pacientes admitidos em uma UTI, 32,7\% apresentavam doença cardiovascular e progrediram para um quadro de sepse e injuria renal aguda. Neste mesmo estudo, que apresentou uma amostra com 564 indivíduos, 25,5\% apresentaram IRA adquirida e destes aqueles com idade avançada apresentam maior risco de desenvolver IRA devido às doenças crônicas como a hipertensão arterial sistêmica e diabetes.

Lopes et al. (2018), ao realizarem uma análise documental em um estudo transversal, descritivo e retrospectivo, elegeram 77 prontuários de pacientes segundo os critérios de inclusão, que estiveram internados no período de janeiro de 2015 a dezembro de 2016. A amostra mostrou-se prevalentemente masculina e foram encontrados como principais fatores de risco para IRA a hipertensão arterial sistêmica (36,36\%) e a diabetes mellitus (20,78\%). Todos estes achados demonstram que as doenças de bases mais prevalentes no país e no mundo são importantes fatores de risco para o desenvolvimento da injuria renal aguda e doença renal crônica.

Acrescenta-se ainda que fatores associados às disfunções hemodinâmicas e metabólicas, como anemia, desnutrição, alterações do metabolismo do cálcio e fósforo podem intensificar a probabilidade de aumento das complicações por doenças cardiovasculares, a disfunção renal e a cronificação desta última (Melo et al., 2015). A elevada prevalência e a crescente incidência destas doenças também são justificadas pela literatura pelo aumento de pessoas com sobrepeso e obesidade em nível mundial, assim como as contribuições advindas das transições epidemiológicas e demográficas também existentes. Logo, o aumento do número de doenças crônicas não transmissíveis, como hipertensão e diabetes, possui um potencial de causar um impacto significativo na morbimortalidade dos pacientes com doença renal (Glodowski \& Wagener, 2015; Hoste et al., 2015).

O diagnóstico inicial de sepse foi verificado neste estudo em aproximadamente $22 \%$ dos prontuários analisados. Dados epidemiológicos trazem a sepse como um dos principais fatores precipitantes de IRA, ocorrendo em cerca $23 \%$ dos pacientes com sepse grave e $51 \%$ daqueles com choque séptico e presença de hemocultura positiva. Estas combinações estão associadas à uma expressiva taxa de mortalidade, em torno de 70\%(Guedes et al., 2017; Okamoto et al., 2012).

Quanto à injúria renal aguda, está apresenta diferentes etiologias e mecanismos amplamente conhecidos, inclusive de formas combinadas. Apresenta o potencial para causar múltiplos danos, como desequilíbrio hemodinâmico, choque séptico e choque nefrotóxico. A lesão renal aguda séptica é geralmente considerada uma consequência global ou regional de hipoperfusão causada por dano tecidual por isquemia-reperfusão. Estratégias de prevenção incluem otimização hemodinâmica, manejo dos fluidos dos pacientes com choque séptico, diminuição do uso inapropriado de drogas nefrotóxicas, como antibióticos e medicamentos de contraste(Hoste et al., 2015).

Quanto às vias de acessos para o tratamento dialítico, é a elevada prevalência de pacientes que chegam aos serviços de diálise em situação de urgência, necessitando de implantação do acesso venoso rápido, caracterizado pela inserção do CTL e CDL. Esta condição muitas vezes mantem o paciente na modalidade de hemodiálise. Gomes et al. (2020) em uma revisão integrativa verificou que o desfecho clínico mais comum nos pacientes em urgência dialítica intra hospitalar é o óbito, consequência principal do ineficiente controle das principais doenças de base causadoras da doença renal, assim como das dificuldades do diagnóstico oportuno dos estágios iniciais pré dialíticos, interferindo, portanto, no prognóstico da doença e tempo de internação do paciente.

O estudo apresentou como limitações: o curto período para seleção de prontuários elegíveis para análise, apenas um pesquisador responsável pela coleta de dados e um corte transversal. Apesar de ser uma amostra modesta (58 prontuários) coletada em um hospital de médio porte, os resultados obtidos deste estudo são importantes, na medida em que apontam para as questões que devem ser refletidas por gestores hospitalares, assim como pelos profissionais responsáveis pelos cuidados em 
saúde e na melhoria da assistência à população acometida pela doença renal. Também contribui com dados importantes ao elucidar o reconhecimento das doenças de base importantes para o surgimento da doença renal em ambiente intra hospitalar a fim de proporcionar um desfecho positivo aos pacientes ainda não submetidos à diálise, impactando nas melhores taxas de sobrevida e redução de complicações nestas internações.

\section{Considerações Finais}

Através da análise do quadro dos pacientes e a situação pregressa de saúde, este estudo mostrou que comorbidades como a hipertensão e o diabetes são fatores diretamente relacionados com o acometimento renal no ambiente intra-hospitalar. Analisar os fatores de influência contribui diretamente no manejo de pacientes, auxiliando assim a equipe de saúde na prevenção de quadros mais complicados de lesão renal, que oportunizam a necessidade de terapia renal substitutiva.

Com isso, vê-se que esse estudo pode alertar para diagnósticos intra-hospitalares comuns que levaram os pacientes a desenvolveram lesão renal aguda. $\mathrm{O}$ conhecimento desses diagnósticos auxilia as equipes de saúde a prestarem serviço mais especializado a beira-leito, contribuindo assim com medidas preventivas e de manutenção da função renal de pacientes internados nas mais diversas unidades de internação.

Assim, se espera que este estudo auxilie em maiores investigações das causas de lesão renal intra-hospitalar. Sendo necessárias investigações de associação da lesão com o uso de medicamentos, tempo de internação hospitalar e em unidade de terapia intensiva, uso de suporte ventilatório, incidência e agentes causadores de sepse, entre outros fatores que potencializam os pacientes internados a precisarem de terapia renal substitutiva.

\section{Referências}

Albuquerque, E. A. Y., Albuquerque, G. de A., Souza, L. C. de, Santos, S. S. dos, \& Rêgo, Y. L. S. (2017). Prontuário eletrônico do paciente e certificação de software em saúde: avanços que visam maior segurança dos dados médicos. Rev. Bras. Inov. Tecnológica Em Saúde

Araújo, A. T. M., Rechmann, I. L., \& Magalhães, T. A. (2019). O sigilo do prontuário médico como um direito essencial do paciente: uma análise a partir das normativas do Conselho Federal de Medicina. Cad. IBERO-Am. Direito sanitário 8, 95-109.

Bacallao Mendez, R. A., Mañalich Comas, R., \& Galvizu Díaz, K. (2016). Estimation of kidney function. In: Fisiologia e exploração funcional renal. 197-199. Havana: Editorial de Ciências Médicas;

Brasil (2018). LEI No 13.787, de 27 de dezembro de 2018. Dispõe sobre a digitalização e a utilização de sistemas informatizados para a guarda, o armazenamento e o manuseio de prontuário de paciente.

Carlino, C., Ávila, R., Monje, L., Mantello, C., Taurizano, N., Urretavizcaya, V., Costantini, M. S., Ferragut, N., Fernández, M. Rosa, G. D. (2020). Primer estudio epidemiológico de injuria renal aguda con requerimiento de terapia de reemplazo renal en Argentina. Sobrevida general y renal a mediano plazo. Rev. Nefrol. Diálisis Traspl. 40, 200-209.

CFM (2002). Conselho Federal de Medicina. Resolução CFM No 1638/2002.

CFM (2007). Conselho Federal de Medicina. Resolução CFM No 1821/2007.

Dias, E. C., Silva, N. A. D., Maia, S. F., Morais, F. F. D., Silva, R. S. D. S., \& Oliveira, L. S. (2017). Avaliação dos indices de infecção relacionados ao cateter duplo lúmen para hemodialise antes e após orientação para o autocuidado. Rev. UNINGÁ 53.

Fernandes, D., Zanelli, T. L. P., Rodrigues, A.S., Rodrigues, M. P., Lodi, J. C., Marques, T. M., Reis, G. dos, Ferreira, L. R. C., \& Milagres, C. S. (2020). Qualidade de vida de pacientes em terapia renal substitutiva: uma análise da doença renal crônica e perfil populacional de risco. Rev. Eletrônica Acervo Saúde $12, \mathrm{e} 4759-\mathrm{e} 4759$

Fernandes, M. I. da C. D., Silva, P. K. de A., Dantas, A. L. de M., Paiva, M. G. M. N. de, Araújo, M. G. de A., \& Lira, A. L. B. de C. (2015). Pacientes em hemodiálise com diagnóstico de enfermagem volume de líquidos excessivo: aspectos socioeconômicos e clínicos. Cogitare Enferm. 20.

Gaspari, M. L. G. de, Helaehil, L. V., Palmieri, G. A., Magalhães, N. S., \& Milagres, C. S. (2019). Prevenção da Nefropatia induzida por contraste em pacientes submetidos a procedimentos em cardiologia intervencionista e hemodinâmica. Saúde Em Rev. 19, 77-86.

Glodowski, S. D., \& Wagener, G. (2015). New insights into the mechanisms of acute kidney injury in the intensive care unit. J. Clin. Anesth. 27, 175-180.

Gomes, Y., Carneiro, R. R., Boução, D. M. N., Moreira, L. C. de S., Sena, E. M. M. \& Oliveira, L. L. (2020). Desfecho clínico de pacientes em urgência dialítica: uma revisão integrativa da literatura/Clinical outcome of patients in dialysis urgency: an integrative literature review. Ciênc. Cuid. E Saúde 19. 
González-Milán, Z. C., Escalona-González, S. O., \& Pavón-Rojas, A. J. (2020). Biomarcadores en la injuria renal aguda. Rev. Electrónica Dr Zoilo E Mar. Vidaurreta 45 .

Guedes, J. da R., Silva, E. S. da, Carvalho, I. L. do N., \& Oliveira, M. D. de (2017). Incidência e fatores predisponentes de insuficiência renal aguda em unidade de terapia intensiva. Cogitare Enferm. 22.

Hoste, E. A. J., Bagshaw, S. M., Bellomo, R., Cely, C. M., Colman, R., Cruz, D. N., Edipidis, K., Forni, L. G., Gomersall, C. D.\& Govil, D. (2015). Epidemiology of acute kidney injury in critically ill patients: the multinational AKI-EPI study. Intensive Care Med. 41, 1411-1423.

Lopes, D., Schran, L. da S., Oliveira, J. L. C. de, Oliveira, R. B. S. R., \& Fernandes, L. M. (2018). Fatores de risco/causais para insuficiência renal aguda em adultos internados em terapia intensiva. Enferm. Bras. 17, 336-345.

Malta, D. C., Bernal, R. T. I., Lima, M. G., Araújo, S. S. C. de, Silva, M. M. A. da, Freitas, M. I. de F., Barros, M. B. de A., Malta, D. C., Bernal, R. T. I.; Lima, M. G. (2017). Noncommunicable diseases and the use of health services: analysis of the National Health Survey in Brazil. Rev. Saúde Pública 51.

Marcus, M. T, \& Liehr P. R. (2001). Abordagens de pesquisa qualitativa. In: Lobiondowood G, Haber J. Pesquisa em Enfermagem: métodos, avaliação crítica e utilização. 4 ed. Guanabara-Koogan, p. 122-139.

Melo, W. F., Pereira, A. W. R., Alves, V. Q., Saldanha, H. G. A. C., \& Sousa, J. da S. (2015). Assistência de enfermagem na urgência e emergência ao paciente vítima de Insuficiência Renal Aguda: uma revisão bibliográfica. Rev. Bras. Educ. E Saúde 5, 06-11.

Neves, P. D. M. de M., Sesso, R. de C. C., Thomé, F. S., Lugon, J. R., \& Nasicmento, M. M. (2020). Brazilian Dialysis Census: analysis of data from the 20092018 decade. Braz. J. Nephrol.

Okamoto, T. Y., Dias, J. C.Y., Taguti, P., Sacon, M. F., Kauss, I. A. M., Carrilho, C. M. D. de M., Cardoso, L. T. Q., Grion, C. M. C., \& Matsuo, T. (2012). Acute renal injury in patients with severe sepsis: prognostic factors. Sci. Medica 22, 138-141.

Patrício, C. M., Machiavelli, J. L., Maia, M. M. \& Navaes, M. de A. (2011). O prontuário eletrônico do paciente no sistema de saúde brasileiro: uma realidade para os médicos? Sci Med 21 .

Pinho, N. A. de, Silva, G. V. da, Pierin, A. M. G., Pinho, N. A. de, Silva, G. V. da, \& Pierin, A. M. G. (2015). Prevalência e fatores associados à doença renal crônica em pacientes internados em um hospital universitário na cidade de São Paulo, SP, Brasil. Braz. J. Nephrol. $37,91-97$.

Ponce, D., Zorzenon, C. de P. F., Santos, N. Y. dos, Teixeira, U. A., \& Balbi, A. L. (2011). Injúria renal aguda em unidade de terapia intensiva: estudo prospectivo sobre a incidência, fatores de risco e mortalidade. Rev. Bras. Ter. Intensiva 23, 321-326.

Ribeiro, W. A., Andrade, M., Fassarella, B. P. A., Santana, P. P. C., Costa, P. A. F. da S., \& Morais, M. C. de (2018). Enfermeiro protagonista na educação em saúde para o autocuidado de pacientes com doenças renal crônica. Rev. Pró-UniverSUS 9, 60-65.

Rodríguez-Palomares, J. R., García, F. L., Cañamas, M. T. T., \& de Arriba, G. (2015). Fracaso renal agudo. Concepto, definición, epidemiologia, etiopatogenia y clasificación, manifestaciones clínicas, complicaciones y diagnóstico diferencial. Medicine. - Programa Form. Médica Contin. Acreditado 11, $4842-4851$.

Schütz, D. M., \& Oliveira, D. S. de (2020). Prontuário eletrônico: uma visão histórica interdisciplinar. 17-32. Revista Universo Psi Taquara.

Stochero G, Sachetti A, Zanettini A, Biasuz S, Reinheimer I. C., \& Carretta, M. B. (2020). Prevalência de nefropatia induzida por contraste em pacientes cardíacos em um setor hemodinâmico. R. pesq.: cuid. fundam.12: 426-431

Thomé, F. S., Sesso, R. C., Lopes, A. A., Lugon, J. R., \& Martins, C. T. (2019). Brazilian chronic dialysis survey 2017. Braz. J. Nephrol.

Vanmassenhove, J., Kielstein, J., Jörres, A., \& Biesen, W. V. (2017). Management of patients at risk of acute kidney injury. The Lancet 389, $2139-2151$. 